\title{
On-Line HEV Energy Management Using a Fuzzy Logic
}

\author{
Yacine Gaoua* $^{* \dagger}$, Stéphane Caux*, Pierre Lopez ${ }^{\dagger \ddagger}$ and Josep Domingo Salvany ${ }^{\S}$ \\ *LAPLACE UMR 5213 CNRS, INPT, UPS, 2 rue Camichel, 31071 Toulouse, France \\ Email: gaoua,caux@laplace.univ-tlse.fr \\ ${ }^{\dagger}$ CNRS, LAAS, 7 avenue du colonel Roche, F-31400 Toulouse, France \\ $\ddagger$ Univ de Toulouse, LAAS, F-31400 Toulouse, France \\ Email: ygaoua,lopez@laas.fr \\ $\S$ Nexter Electronics, 6 rue Claude-Marie Perroud, F-31047 Toulouse, France \\ Email: j.domingo@nexter-group.fr
}

\begin{abstract}
This paper presents different methods and approaches allowing a better energy management for Hybrid Electrical Vehicle presenting a multi-source system, in order to increase its autonomy, reduce the costs of its energy consumption and decrease pollutant emission. The main goal sought is to minimize the battery discharge allowing the vehicle to make a maximum number of cycles (repeating the same mission), while respecting constraints related to the energy system functioning and design of both sources. To do this, a fuzzy approach is proposed, and tuned using an evolutionary algorithm, to finally manage on-line the dispatching of electrical energy minimizing the consumption criterion thus increasing vehicle autonomy. To measure the quality of the on-line solution, off-line study is realized on a known mission profile and optimization based on a nonlinear modeling of the problem due to the sources characteristics is used. The problem is solved using optimization techniques which give a good global optimum in order to compare it with the battery discharge obtained on-line.
\end{abstract}

\section{INTRODUCTION}

Hybrid Electrical Vehicles (HEV) consist of at least two energy sources onboard (batteries, supercapacitors and/or fuel cells) of different characteristics (efficiency, energy and power). The reversible sources like batteries or supercapacitors can store energy when the vehicle brakes and provide it in traction phases. However, it is of paramount importance to find a smart splitting strategy to minimize the battery discharge considered as the main source of power and to satisfy the instantaneous power demand of the powertrain while respecting the different constraints of functioning, design and security in order to minimize the batteries discharges on the overall mission.

In the first part of this paper, applying fuzzy logic allows us to find a real time sub-optimal solution. Using this method requires a certain expertise level about the energy system behavior in order to tune the fuzzy algorithm. To circumvent this difficulty, application of an evolutionary algorithm such as a Genetic Algorithm (GA) appears an effective way to adjust off-line parameters of the fuzzy algorithm on known-mission profiles. This allows light on-line computation and an accurate decision due to off-line optimization [1].

The second part of the paper is devoted to quality evaluation of the on-line solution obtained using fuzzy logic. An off-line

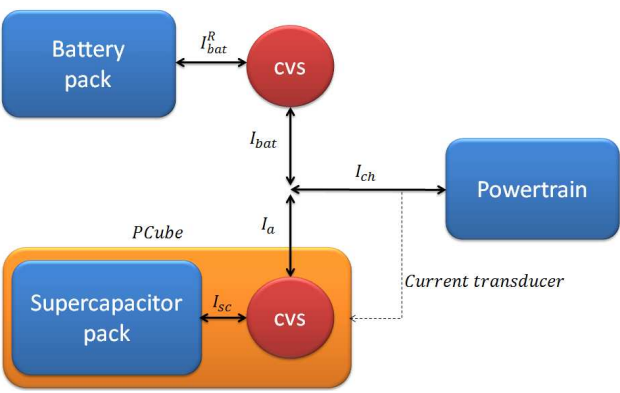

Fig. 1. Vehicle energy chain.

optimization is carried out on a known-mission profile using optimization techniques of COIN-OR methods (The COmputational INfrastructure for Operations Research) [2] such as IPOpt (Interior Point Optimizer) [3] or BONMIN (Basic Opensource Nonlinear Mixed INteger programming) [4] applied to the nonlinear modeling of the problem. Therefore, taking into account the future demands of the powertrain, allows the optimization to give a good local optimum (near the global one) that will be compared with the on-line solution obtained.

\section{DESCRIPTION OF THE ENERGY CHAIN}

The energy chain of the vehicle concerned consists of two energy sources (Figure 1). A battery pack connected to the distribution bus via a bidirectional converter, and a subsystem (PCube) allowing the assistance of the battery pack in the management of energy transfer.

The PCube contains a pack of supercapacitors connected to the network via a bidirectional converter, several safety and measuring devices to control current limits and measure the supercapacitor voltage. The consumption source is represented by a powertrain which provides positive power demand when the vehicle is in traction and negative power during braking phases.

The converter is a power electronic module which delivers a current maintaining a regulated output voltage. It keeps the bus voltage to its reference despite voltage variations of the battery pack and the supercapacitors pack. It is characterized 


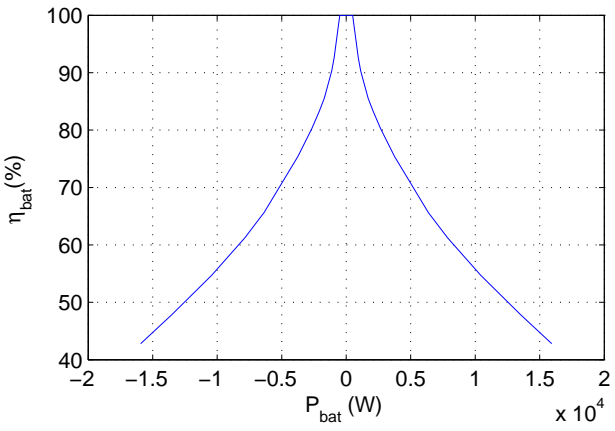

Fig. 2. Battery efficiency.

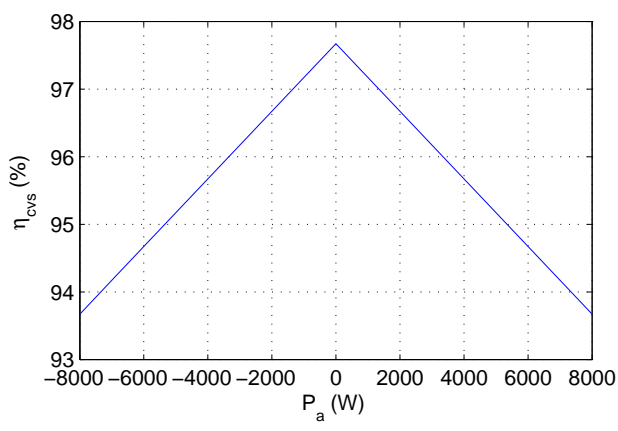

Fig. 3. Chopper efficiency of the PCube pack.

by high efficiency from $93 \%$ up to $97 \%$ due to the high quality of power electronic components and internal control laws.

\section{A. Battery Efficiency}

The battery pack efficiency is computed from the efficiency of the battery itself and the efficiency of its converter included. According to the experiments conducted by Nexter Electronics, the battery efficiency $\eta_{b a t}()$ decreases by increasing its out power $P_{b a t}$ causing large energy losses, as shown in Figure 2.

From the battery efficiency, its energy losses Eloss bat can be derived using the following formulations:

$$
\begin{array}{ll}
P_{b a t}^{R}=\frac{P_{b a t}}{\eta_{b a t}\left(P_{b a t}\right)} & P_{b a t} \geq 0 \\
P_{b a t}^{R}=P_{b a t} \eta_{b a t}\left(P_{b a t}\right) & P_{b a t}<0 \\
\text { Eloss bat }_{\text {blo }}=\left|P_{\text {bat }}^{R}-P_{\text {bat }}\right| &
\end{array}
$$

where $P_{b a t}^{R}$ corresponds to its real power provided or recovered.

\section{B. PCube Static converter Efficiency}

In the same way, the computation of the supercapacitor pack energy losses is meanly based on the current passing through its internal resistance. It is interesting to focus on energy losses of the PCube convertor Eloss cvs which are calculated in function of its efficiency $\eta_{c v s}()$ and its out power $P_{a}$ (Figure
3) using the following formulation:

$$
\begin{array}{ll}
P_{s c}=\frac{P_{a}}{\eta_{c v s}\left(P_{a}\right)} & P_{a} \geq 0 \\
P_{s c}=P_{a} \eta_{c v s}\left(P_{a}\right) & P_{a}<0 \\
\text { Eloss }_{c v s}=\left|P_{a}-P_{s c}\right| &
\end{array}
$$

where $P_{s c}$ corresponds to the power provided/recovered by the supercapacitor pack.

\section{Mathematical Modeling}

The goal is to minimize the battery discharge in order to meet the power demand of the powertrain at each instant, while respecting the different constraints of functioning, safety and design. A mathematical model is developed reflecting the optimization problem. The meaning of the mathematical model is as follows:

- (7) The demand of the powertrain must be satisfied by both sources, when the vehicle is in traction (i.e., $I_{c h} \geq$ $0)$,

- (8) Recovering all braking energy in the capabilities limits of the two sources when $I_{c h}<0$ ),

- $(9,10)$ Safety constraint represented by $\mathrm{min} / \max$ current limits of the PCube,

- $(11,12)$ Storage capacity of the two sources (state of charge for batteries, voltage for supercapacitors),

- $(13,14)$ Energy losses of the battery and the PCube,

- $(15,16)$ State of Charge evolution of the two sources,

- (17) Computation of the battery voltage evolution.

Considering $E_{b a t} \Delta t$ corresponds to the electrical quantity provided or recovered by the battery, and $f$ the function deducing the battery voltage knowing its initial State of Charge $\operatorname{Soc}_{\text {bat }}(0)$.

Input parameters are defined in Table I. Consequently, the decision variables of the model are:

- $I_{b a t}$ Current provided or recovered by the battery,

- $I_{b a t}^{R}$ Real current provided or recovered by the battery,

- $I_{a}$ Current entering or exiting the PCube,

- $I_{s c}$ Current provided or recovered by the supercapacitor,

- $U_{b a t}$ Supercapacitor voltage,

- $S o c_{b a t}$ State of Charge of the battery.

$$
\begin{aligned}
& I_{a}+I_{b a t}=I_{c h} \\
& I_{c h} \leq I_{a}+I_{b a t} \leq 0 \\
& I_{s c}^{\text {min }} \leq I_{s c} \leq I_{s c}^{\max } \\
& I_{a}^{\text {min }} \leq I_{a} \leq I_{a}^{\text {max }} \\
& S O C_{b a t}^{\text {min }} \leq S O C_{b a t} \leq S O C_{b a t}^{\text {max }} \\
& U_{s c}^{\text {min }} \leq U_{s c} \leq U_{s c}^{\text {max }} \\
& P_{b a t}^{R}=P_{b a t}+\operatorname{Eloss}_{b a t}\left(P_{b a t}\right) \\
& P_{s c}=P_{a}+\operatorname{Eloss}_{c v s}\left(P_{a}\right)+R_{s c}\left(I_{s c}\right)^{2} \\
& S O C_{b a t}=S O C_{b a t}(0)-\left(E_{b a t} / C_{a p} p_{b a t}\right) \Delta t \\
& U_{s c}=U_{s c}(0)-\left(I_{s c} / C_{s c}\right) \Delta t-\left(R_{s c} I_{s c}\right) \\
& U_{b a t}=f\left(\operatorname{Soc}_{b a t}(0)\right)
\end{aligned}
$$


TABLE I

INPUT PARAMETERS.

\begin{tabular}{|c||c|}
\hline Parameters & Meaning \\
\hline$I_{c h}$ & Demand of the powertrain \\
\hline$I_{a}^{\text {max }}$ & Maximum current exiting the PCube converter \\
\hline$I_{a}^{\text {min }}$ & Minimum current exiting the PCube converter \\
\hline$I_{s c}^{\max }$ & Maximum current provided by the supercapacitor \\
\hline$I_{s c}^{\text {min }}$ & Minimum current provided by the supercapacitor \\
\hline$U_{s c}^{\text {max }}$ & Maximum voltage of the supercapacitor \\
\hline$U_{s c}^{\text {min }}$ & Minimum voltage of the supercapacitor \\
\hline$U_{s c}(0)$ & Initial voltage of the supercapacitor (initial charge) \\
\hline$S o c_{b a t}^{\text {max }}$ & Maximum energy level allowed in the battery pack \\
\hline$S o c_{b a t}^{\min }$ & Minimum possible energy level in the battery \\
\hline$S o c_{b a t}(0)$ & Initial energy level in the battery \\
\hline$C_{a p_{b a t}}$ & Battery capacity \\
\hline$\Delta t$ & Time stepsize \\
\hline$R_{s c}$ & Supercapacitor internal resistance \\
\hline$C_{s c}^{\text {max }}$ & Supercapacitor capacity \\
\hline$E l o s s_{b a t}$ & Battery energy losses \\
\hline$E l o s s_{c v s}$ & \\
\hline & Energy losses of the PCube converter \\
\hline
\end{tabular}

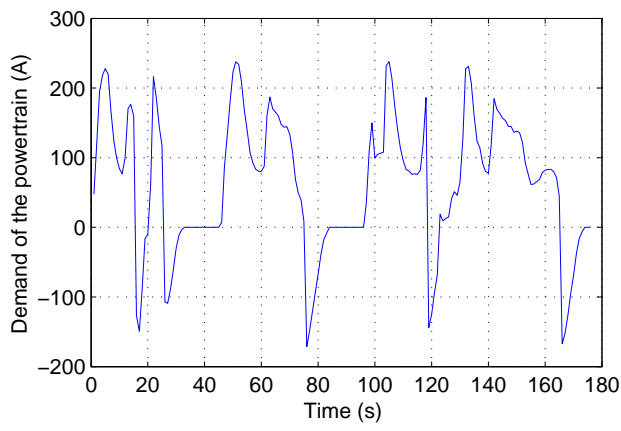

Fig. 4. Nexter Electronics mission profile.

\section{HEV Energy Management}

A mission profile is proposed in Figure 4, which corresponds to the instantaneous power demand of an electric vehicle provided by Nexter Electronics. The energy management policy should minimize the battery discharge for such mission while satisfying system constraints.

\section{A. Fuzzy Logic Method}

The theoretical bases of Fuzzy Logic (FL) [5][6] are established so as to be able to treat inaccurate variables of values between 0 and 1, according to their membership degrees in the verification of a condition, contrary to Boole's logic in which variables must take values 0 or 1 . It is particularly adapted in the case of on-line energy management where there are uncertainties following an actual mission profile. The FL is an on-line method composed of three steps: Fuzzification, Rules engine, and Defuzzification. The solution given by this method is suboptimal because the optimization is instantaneous and does not take into account future requests. Difficulty consists in adjusting FL tuning parameters off-line. Application of an

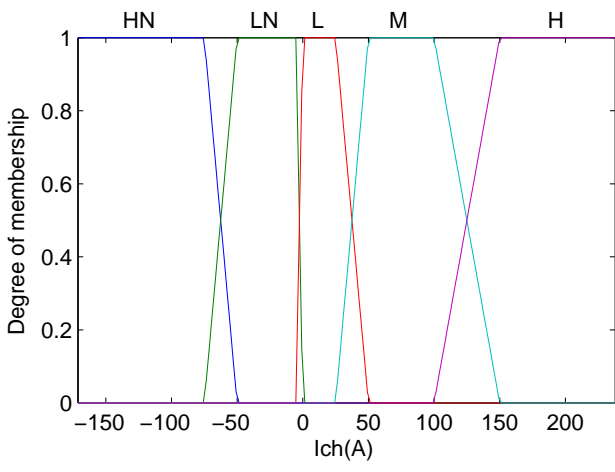

Fig. 5. Demand of the powertrain.

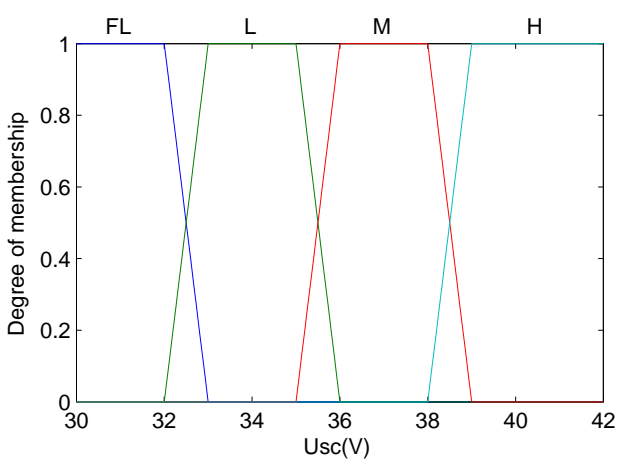

Fig. 6. Supercapacitor voltage.

evolutionary algorithm (e.g., Genetic Algorithm) permits the adjustment of the membership functions parameters.

The Fuzzification is the first step of the fuzzy processing. It consists in defining the linguistic variables and the membership functions which can take the form of a triangle, a trapezoid, or gaussian functions. For flexibility and low complexity reasons in on-line computations, the chosen functions take trapezoidal shape.

The fuzzy system applied on the PCube model contains three variables: two input variables which represent the demand of the powertrain $\left(I_{c h}\right)$ and the supercapacitor voltage $\left(U_{s c}\right)$, and one output variable corresponding to the current provided or recovered by the battery $\left(I_{b a t}\right)$. Each variable is defined by their membership functions chosen according to the expertise level of the decision maker, as shown in Figures 5, 6 and 7.

Each membership function has a name describing the state of the variable: $I_{c h} \in\{H N, L N, L, M, H\}, U_{s c} \in$ $\{F L, L, M, H\}$ and $I_{b a t} \in\{H N, L N, Z, L, M, H\}$ with $\mathbf{H N}$ High Negative, LN Low Negative, $\mathbf{Z}$ Nil, FL Fairly Low, L Low, $\mathbf{M}$ Medium and $\mathbf{H}$ High.

The second step is the rules engine which permit the link between the input and the output variables using the operators $I F, A N D, O R$ to draw conclusions. Table II summarizes all possible situations of the battery functioning for each variation of the supercapacitor voltage and the demand of the powertrain, as shown in Figure 8. 


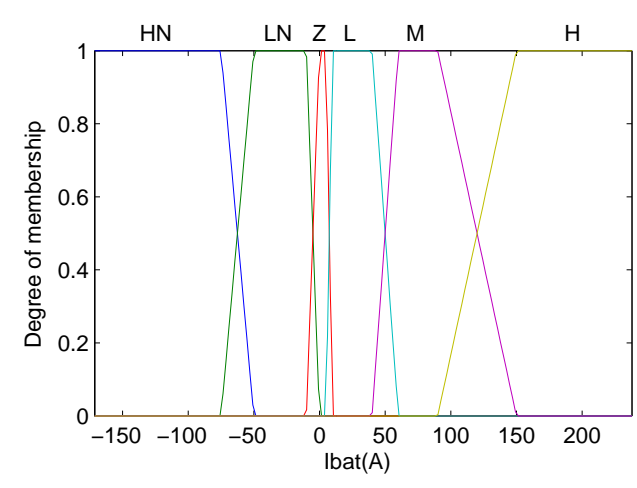

Fig. 7. Battery current.

TABLE II

RULES ENGINE.

\begin{tabular}{|c|c|c|c|c|}
\hline$\left(I_{c h}, U_{s c}\right)$ & $\mathbf{F L}$ & $\mathbf{L}$ & $\mathbf{M}$ & $\mathbf{H}$ \\
\hline $\mathbf{H N}$ & $\mathrm{Z}$ & $\mathrm{Z}$ & $\mathrm{LN}$ & $\mathrm{HN}$ \\
\hline $\mathbf{L N}$ & $\mathrm{Z}$ & $\mathrm{Z}$ & $\mathrm{Z}$ & $\mathrm{LN}$ \\
\hline $\mathbf{L}$ & $\mathrm{L}$ & $\mathrm{L}$ & $\mathrm{L}$ & $\mathrm{Z}$ \\
\hline $\mathbf{M}$ & $\mathrm{M}$ & $\mathrm{M}$ & $\mathrm{L}$ & $\mathrm{Z}$ \\
\hline $\mathbf{H}$ & $\mathrm{H}$ & $\mathrm{M}$ & $\mathrm{L}$ & $\mathrm{Z}$ \\
\hline
\end{tabular}

For each input value assigned to the supercapacitor voltage and the demand of the powertrain, the rules engine generates more than four rules when the values belong to the fuzzy set defined by the membership functions intersection.

For example, if $\left(I_{c h}=125 \mathrm{~A}\right)$ and $\left(U_{s c}=32.25 \mathrm{~V}\right)$ the possible situations are: $I_{c h}=M$ (resp. $H$ ) with $50 \%$ (resp. $50 \%$ ) of probability, $U_{s c}=F L$ (resp. $L$ ) with $75 \%$ (resp. $25 \%$ ) of probability, and the rules engine generates the following rules using the operators $A N D$ (resp. $O R$ ) corresponding to the minimum (resp. the maximum) applied to the membership functions of the two variables $I_{c h}$ and $U_{s c}$ :

- If $\left(I_{c h}=H\right)$ and $\left(U_{s c}=F L\right)$ then $\left(I_{b a t}=H\right)$ or

- If $\left(I_{c h}=H\right)$ and $\left(U_{s c}=L\right)$ then $\left(I_{b a t}=M\right)$ or

- If $\left(I_{c h}=M\right)$ and $\left(U_{s c}=F L\right)$ then $\left(I_{b a t}=M\right)$ or

- If $\left(I_{c h}=M\right)$ and $\left(U_{s c}=L\right)$ then $\left(I_{b a t}=M\right)$.

The final step is the Defuzzification. It consists in computing the abscissa of the output variable $I_{c h}$ using centroid method on the resulting fuzzy set. By applying the fuzzy logic on the Nexter mission profile previously defined, the battery discharge unregistered is $2.98 \%$.

Figure 8 represents the possible decisions surface obtained on the Nexter mission profile allowing to give the current provided by the battery for each variation of supercapacitor voltage and the demand of the powertrain.

Parameters setting of the membership functions depends essentially on the perfect knowledge of the system and the expertise level. To circumvent this problem, a genetic algorithm was used to optimize and adjust off-line the parameters of each membership function on reference mission profiles, in order to obtain an accurate and an adequate solution.

After the off-line setting of the membership functions of each variable with GA and application of the fuzzy logic on

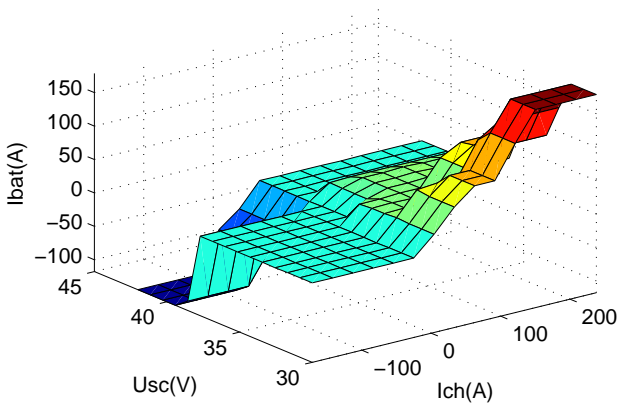

Fig. 8. Decision surface.

\begin{tabular}{|c|c|}
\hline & rithm 1 Genetic algorithm. \\
\hline & ire: Random choice of an initial population \\
\hline & vhile Stopping criterion not achieved do \\
\hline 2: & for Each individual in the population do \\
\hline 3: & while The mission is not over do \\
\hline 4: & Building of the fuzzy system \\
\hline 5: & $\begin{array}{l}\text { Deduction of the solution }\left(I_{b a t}, S_{o c} c_{b a}, I_{a}, I_{s c} \text {, }\right. \\
\left.U_{s c}\right) \text { by the fuzzy system }\end{array}$ \\
\hline 6: & Solution checking by the model constraints \\
\hline 7: & if violated constraint then \\
\hline 8: & $\begin{array}{l}\text { Correction of the solution }\left(I_{b a t}, S_{o c} c_{b a t}, I_{a}, I_{s c} \text {, }\right. \\
\left.U_{s c}\right)\end{array}$ \\
\hline 9: & end if \\
\hline 10: & end while \\
\hline 11: & return Battery discharge \\
\hline 12: & end for \\
\hline 13: & Selection of the best individuals \\
\hline & Mutation and crossover (standard law) \\
\hline & Evaluation of the new population \\
\hline 16: & Creation of the new population \\
\hline & d while \\
\hline & eturn Best individual \\
\hline
\end{tabular}

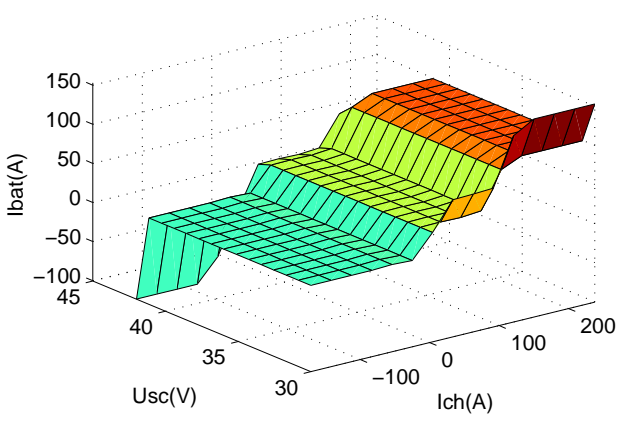

Fig. 9. Decision surface using genetic algorithm.

the Nexter mission profile using Global Optimization and Fuzzy Logic toolbox of Matlab [7][8], the battery discharge is $2.92 \%$, and the decisions surface obtained is shown in Figure 9.

In Figure 10, the battery provides more than it recovers in order to meet the demand of the powertrain and maintaining 


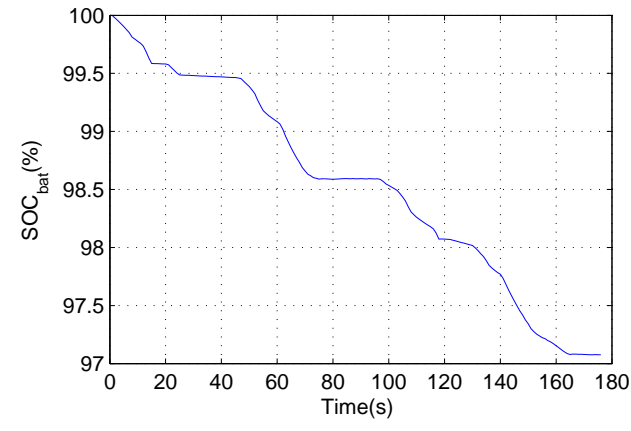

Fig. 10. Battery state of charge.

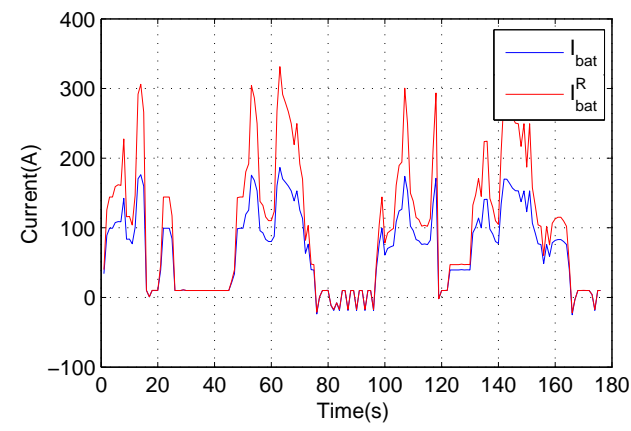

Fig. 11. Current provided/recovered by the battery.

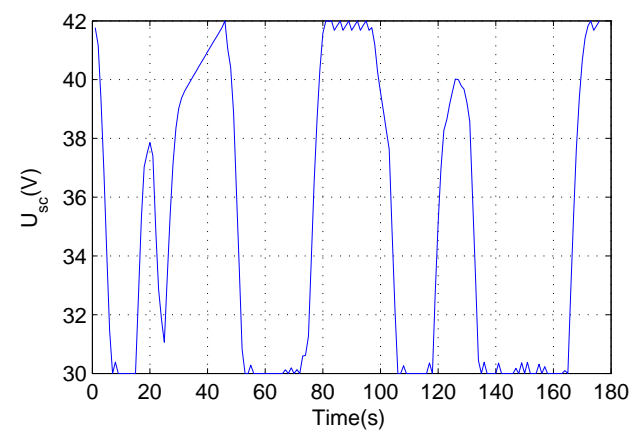

Fig. 12. Supercapacitor voltage.

the supercapacitor voltage between its limits. The poor battery efficiency conduce to recover the majority of the braking energy with the supercapacitor pack. When it reaches its maximum charge level, the battery recovers the rest of the energy thus globally increases the efficiency and autonomy. Variations of the battery current are caused by its energy losses (see Figure 11).

The limits constraints of the PCube current and the supercapacitor voltage are respected, as shown in Figures 12 and 13. The high supercapacitor efficiency allows us to recover energy (negative current) from the braking phase and provide it when the vehicle is in traction, in order to minimize the battery discharge avoiding as frequently as possible its poor efficiency part. At the end of the mission, the supercapacitor voltage reaches its maximum level due to the recovery of all braking energy during the last braking phases. The power variations

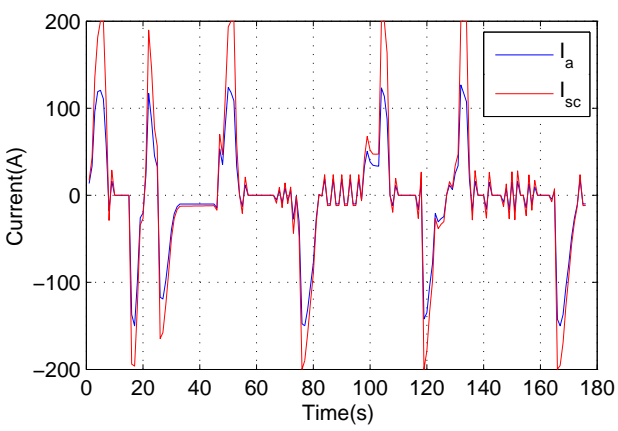

Fig. 13. Current provided/recovered by the PCube.

of the PCube correspond to its energy losses.

\section{B. COIN-OR Methods}

To compare the optimized FL method results obtained, the global minimum consumption should be evaluated. IPOpt (Interior Point Optimizer) and BONMIN (Basic Open-source Nonlinear Mixed INteger programming) are open source software packages of the COIN-OR methods (The COmputational INfrastructure for Operations Research). They are used off-line to find a local optimum of nonlinear problems on a knownmission profile. IPOpt is based on the computation of the gradient and the Hessian of Lagrangian. In order to apply this method, the constraints and the objective function must be twice continuously differentiable.

BONMIN is an experimental open-source $C++$ code for solving nonlinear mixed integer program. It contains several methods of operations research such as Branch-and-Bound based on nonlinear programming, Outer-Approximation decomposition, Quesada and Grossmann's Branch-and-Cut algorithm and a hybrid outer-approximation based on a Branchand-Cut algorithm. This package provides accurate minimum and are not computer time consuming with regard to other offline global optimization algorithms (Dynamic Programming, Optimal Control, etc.)

The objective is to minimize the battery discharge (maximize HEV autonomy), in order to increase the number of cycles to be realized by the vehicle, while respecting the model constraints and the power demand. The objective function is formulated as follows:

$$
\min \left(\operatorname{Soc}_{b a t}^{\max }-\operatorname{Soc}_{b a t}(T)\right) \equiv \max \operatorname{Soc}_{b a t}(T)
$$

Actually the supercapacitor is reloaded by the battery at each stop of the vehicle despite its poor efficiency. To avoid this, and allow the vehicle to carry successive missions, a constraint on the final SoC of the supercapacitor is optionally added in the optimization to reload it in an intelligent manner.

$$
U_{s c}(T)=U_{s c}^{\max }
$$


The global model used by IPOpt is as follows:

$$
\begin{aligned}
& \max S_{\operatorname{coc}_{b a t}}(T) \\
& I_{a}(t)+I_{b a t}(t)=I_{c h}(t) \\
& I_{c h}(t) \leq I_{a}(t)+I_{b a t}(t) \leq 0 \\
& I_{s c}^{\text {min }} \leq I_{s c}(t) \leq I_{s c}^{\max } \\
& I_{a}^{\text {min }} \leq I_{a}(t) \leq I_{a}^{\text {max }} \\
& S O C_{b a t}^{\text {min }} \leq S O C_{b a t}(t) \leq S O C_{b a t}^{\max } \\
& U_{s c}^{\text {min }} \leq U_{s c}(t) \leq U_{s c}^{\text {max }} \\
& P_{b a t}^{R}(t)=P_{b a t}(t)+E l o s s_{b a t}\left(P_{b a t}(t)\right) \\
& P_{s c}(t)=P_{a}(t)+E_{o s s_{c v s}}\left(P_{a}(t)\right)+R_{s c}\left(I_{s c}(t)\right)^{2} \\
& S O C_{b a t}(t)=S O C_{b a t}(t-1)-\left(E_{b a t}(t) / C_{a p} p_{b a t}\right) \Delta t \\
& U_{s c}(t)=U_{s c}(t-1)-\left(I_{s c}(t) / C_{s c}\right) \Delta t-\left(R_{s c} I_{s c}(t)\right) \\
& U_{b a t}(t)=f\left(S_{o c}(t-1)\right) \\
& U_{s c}(T)=U_{s c}^{\text {max }}
\end{aligned}
$$

To determine the number of cycles to be performed by the vehicle according to the discharge depth defined, a program is developed using the AMPL language (Modeling Language for Mathematical Programming) [9] and COIN-OR methods.

The program uses as input parameters, the mathematical model with .mod extension and input data with .dat extension written in AMPL format. The AMPL mathematical tool creates automatically a file with a.$n l$ extension containing the initial solution and information about the Hessian of the Lagrangian. The optimization is launched by IPOpt or BONMIN, using the command ipopt file.nl or bonmin file.nl all included in the solver package.

If the optimization runs in safe mode, the program retrieves the results, increments the number of cycles and updates the data of the problem file.dat. The process is iterative until the optimization stops by detecting an error which indicates that the battery SoC is insufficient to meet the demand of the powertrain for the considered mission.

To compare the results obtained previously using FL, the IPOpt program was launched on one iteration, which corresponds to the realization of one cycle on the Nexter Electronics mission profile. The battery discharge is $2.6 \%$.

\section{CONClusion}

A formal method is proposed using open source optimization package. This off-line computation uses the complete mathematical formulation of the problem and result is considered as comparison value. For on-line energy management a fuzzy logic decision system is used and off-line tuning phase is presented. The optimized Fuzzy Logic decision result obtained on the same mission profile is very close the optimal one. Moreover the optimized FL allows us to also react optimally with uncertainties in the power demand of the mission, reaching the lowest possible consumption. Fuzzy logic allows us to find a real time energy management for the hybrid vehicle concerned, while respecting the different constraints. It can find the current provided by the battery for each variation of the supercapacitor voltage and the demand of the powertrain.
The quality of the solution depends on the membership functions and the rules engine of the fuzzy system. Optimization added using the genetic algorithm has allowed to improve solution quality by adjusting the membership functions offline.

The off-line study realized on a known-mission profile using the COIN-OR methods, confirms the effectiveness of the results obtained by the fuzzy logic method.

\section{REFERENCES}

[1] S. Caux, D. Wanderley-Honda, D. Hissel, and M. Fadel, "On-line energy management for HEV based on particle swarm optimization," The European Physical Journal Applied Physics, vol. 54, pp. 1-9, 2011.

[2] R. Lougee-Heimer, "The common optimization interface for operations research," IBM Journal of Research and Development, vol. 47, pp. 57-66, 2003.

[3] A. Wächter, "Short tutorial: Getting started with ipopt in 90 minutes," in Combinatorial Scientific Computing, ser. Dagstuhl Seminar Proceedings, U. Naumann, O. Schenk, H. D. Simon, and S. Toledo, Eds., no. 09061. Dagstuhl, Germany: Schloss Dagstuhl - Leibniz-Zentrum fuer Informatik, Germany, 2009.

[4] P. Bonami and J. Lee, BONMIN Users Manual, August 2007.

[5] M. Hellmann, Fuzzy Logic Introduction, 2001.

[6] L. A. Zadeh, "Fuzzy sets," Information and Control, vol. 8, pp. 338-353, 1965.

[7] MathWorks, "Global optimization toolbox: Genetic algorithm." [Online]. Available: http://www.mathworks.fr/fr/help/gads/genetic-algorithm.html

[8] —_ "Fuzzy logic toolbox." [Online]. Available: http://www.mathworks. $\mathrm{fr} / \mathrm{fr} / \mathrm{help} /$ fuzzy/mamdani-fuzzy-inference-systems.html

[9] R. Fourer, D. M. Gay, and B. W. Kernighan, "Modeling language for mathematical programming," Management Science, vol. 36, pp. 519-554, 1990. 